\title{
New Implementation of an SX700 Undulator Beamline at the Advanced Light Source
}

\author{
T. Warwick ${ }^{1}$, N. Andresen ${ }^{1}$, J. Comins ${ }^{1}$, K. Kaznacheyev ${ }^{2}$, J. B. Kortright ${ }^{1}$, J. P.

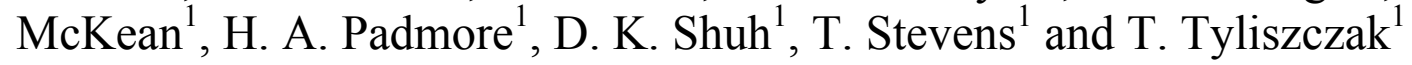 \\ ${ }^{1}$ Lawrence Berkeley National Laboratory, Berkeley, California 94720, USA \\ ${ }^{2}$ Canadian Light Source, Univ. of Saskatchewan, Saskatoon SK S7N OX4, Canada
}

\begin{abstract}
A newly engineered implementation of a collimated SX700-style beam line for soft X-rays is described. This facility is operational at the Advanced Light Source and delivers high brightness undulator beams to a scanning zone plate microscope and to an array of end stations for x-ray spectroscopic studies of wet surfaces. Switching between branches is motorized, servo-steering systems maintain throughput and the monochromator works together with the elliptical undulator for a fully automated facility.
\end{abstract}

\section{INTRODUCTION}

SX700 style monochromators, developed at BESSY [1,2,3], have been adopted for high-resolution soft x-ray spectroscopy at several synchrotron sources. In this paper we describe a new undulator beam line facility employing this monochromator scheme, operating without entrance slits and installed in collimated light. This implementation is a new engineering design that emphasizes cooling and cleanliness. It is a general-purpose facility serving a suite of micro-focus spectroscopy experiments and a diffraction limited zone plate scanning x-ray microscope.

\section{DESIGN FEATURES}

In a beam line on a high power undulator the cooling of the optics becomes a challenge [4]. The new engineering employed in this facility provides optimized cooling, with water introduced through the monochromator scan-drive shafts, to minimize flexing bellows connections. The cooling scheme allows the beam line to operate at maximum efficiency and to deliver zero-order light.

The beam line delivers light at a specific wavelength from each one of two gratings, with widely different line density, so that instruments with limited phase space acceptance (such as the zone plate microscope) can be illuminated at low resolution, using low dispersion to maximize the illumination phase space density, or at high resolution from the finer grating. This trade-off between gratings is particularly important for the $\mathrm{C}, \mathrm{N}$ and $\mathrm{O} 1 \mathrm{~s}$ edges and is made possible by the extended energy range from each grating. This is nice a feature of the SX700 optical scheme. Using a fine grating with maximum efficiency at low energy requires small included angle so that the stringent requirements for monochromator cooling cannot be avoided. The new monochromator has a large silicon pre-mirror, with internal water cooling, and an internally cooled copper grating, wide enough for multiple rulings.

The two toroidal focus mirrors after the monochromator each generate a stigmatic focus, alternatively at the two exit slits. These become illumination sources for the zone plate microscope in one branch, and for the KB refocus mirrors in the other branch. One of these two toroidal mirrors is retractable (by a few $\mathrm{mm}$ ) so that switching from one set of slits to the other is easily automated. The horizontal steering capability of these mirrors is used in a servoloop to lock the focus through the horizontal width of the exit slit aperture, by equalizing photocurrents from the left and right slit blades. This servo loop eliminates horizontal motion of the focal spot due to thermal drift of 
components, due to changes of the horizontal position of the stored beam, and due to residual horizontal steering as the monochromator is scanned. These three sources of horizontal motion are of similar magnitude and are easily compensated with a small amount of steering $(20 \mu \mathrm{rad})$.

Precise reproducibility of the energy scale as the gratings are switched is achieved by a grating change mechanism under the monochromator vessel which moves the chamber transversely with high angular reproducibility. Furthermore, in the vertical (dispersion) plane there is a safety margin in the entrance slit-less optical scheme to allow for vertical motion of the electron beam five times larger than the nominal $(40 \mu \mathrm{m})$ beam size, before the energy scale of the monochromator is changed by more than 1 part in 8000 .

Much of the research planned at this facility will be at the C1s edge. We have tried for a clean assembly to minimize the carbon absorption in the beam line. Facilities such as this can lose up to a factor of 50 in the delivered flux at the carbon edge due to organic contamination. On the other hand, clean lines (at the ALS) can lose no more than $30 \%$. While cleaning techniques exist, it is far better to put together a clean system that stays clean. In the new monochromator design the angle encoders are outside the vacuum so that the design risks small non-linear angle tracking errors for the sake of a simple internal arrangement of parts that can be made clean.

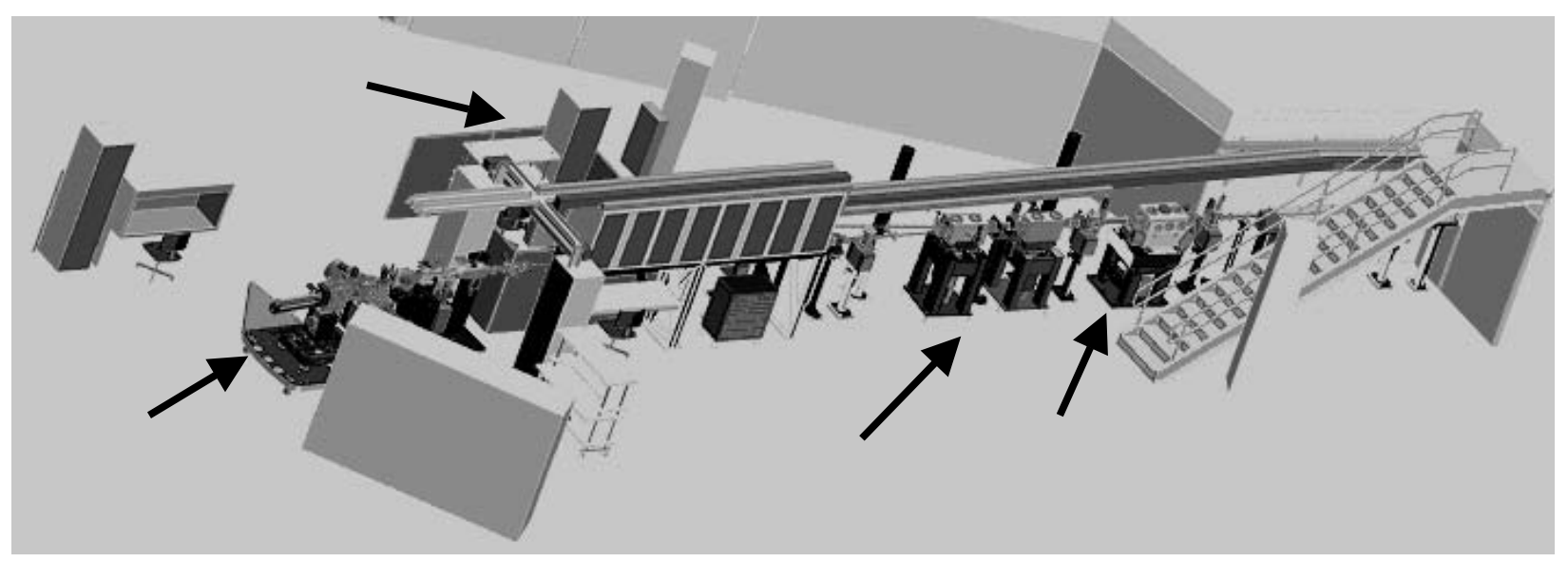

FIGURE 1. Beam line components on the floor of the ALS.

\section{BASIC PERFORMANCE}

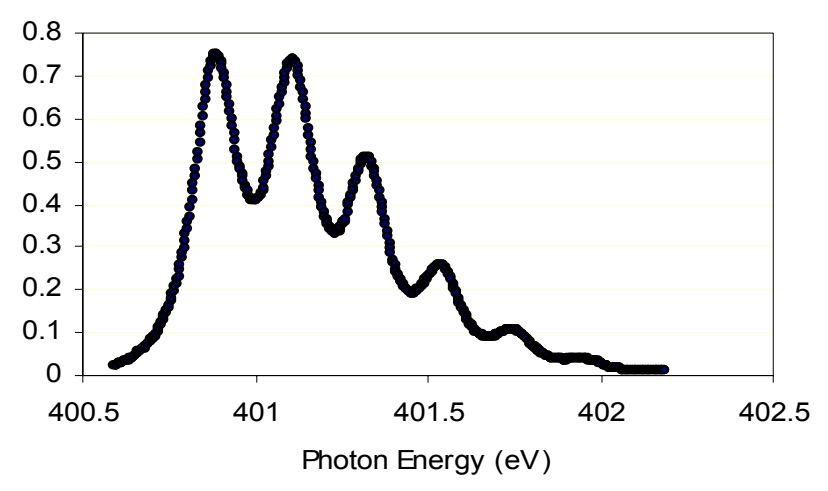

FIGURE 2. $\mathrm{N}_{2}$ absorption, $1 \mathrm{~s}$ to $\pi^{*}$ orbital, with vibrational levels resolved. This spectrum is measured at high resolution $(\mathrm{R}>8000)$ with $\mathrm{Cff}=2$ from the 1200 lines/mm grating and shows the natural line widths.
Figure 2 shows an $\mathrm{N}_{2}$ vibrational spectrum measured by observing nitrogen $1 \mathrm{~s}$ absorption in the air of the STXM vessel. This spectrum is measured from the 1200 lines $/ \mathrm{mm}$ grating at $\mathrm{Cff}=2.2$ ( $\mathrm{Cff}=\cos \beta / \cos \alpha)$. This grating covers the carbon edge and can be used from $280 \mathrm{eV}$ up to $2000 \mathrm{eV}$. It has a platinum optical surface. Energy calibration is achieved using $\mathrm{CO}_{2}, \mathrm{~N}_{2}, \mathrm{O}_{2}, \mathrm{Ne}, \mathrm{Ar}$ and $\mathrm{Kr}$. The reproducibility of the energy scale from scan to scan, and against grating changeover and source motion, is better than $30 \mathrm{meV}$. Figure 3 shows the measured flux (at $400 \mathrm{~mA}$ electron current) delivered through the exit slit, continuously adjusted for resolving power $\mathrm{R}=3000$. These measurements are in very close agreement to the design computations [5]. The 1200 lines $/ \mathrm{mm}$ grating can deliver spectral resolution better than $1: 8000$ at the most efficient value of $\mathrm{Cff}=2.2$. The 150 lines $/ \mathrm{mm}$ grating delivers light at lower resolution $(1: 3500, \mathrm{Cff}=1.6)$ and higher brightness, at the $\mathrm{C}, \mathrm{N}$ and $\mathrm{O}$ edges. At the $\mathrm{C}$ edge it can be operated with variable $\mathrm{Cff}$ so that higher resolution $(1: 4500$ at $\mathrm{Cff}=4)$ is available at reduced efficiency. The higher values of Cff provide higher resolution because of increased dispersion and, more importantly, because of decreased sensitivity to optical slope errors, from polishing and from thermal effects. 


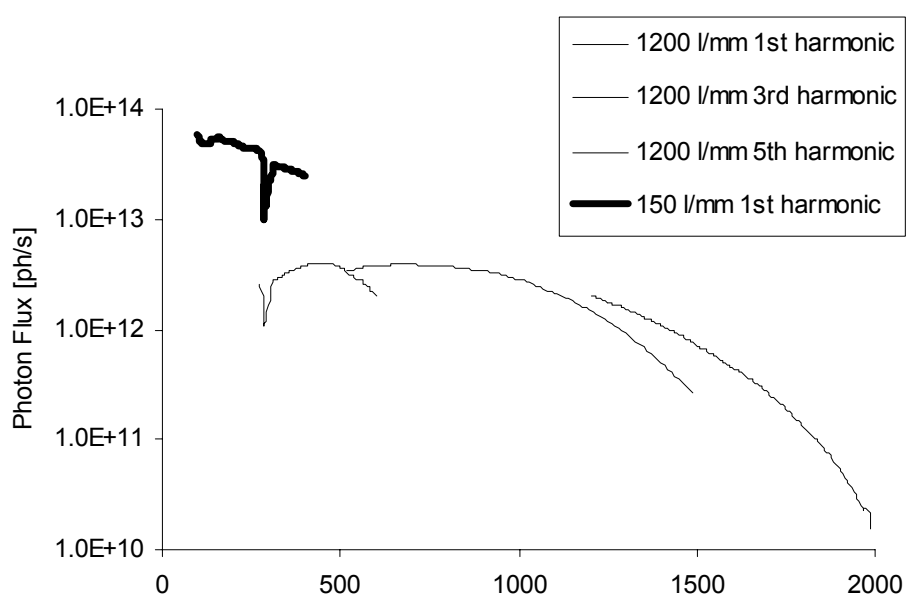

FIGURE 3. Photon flux measured for $E / \delta E=3000$

The variable polarization of the EPU was measured directly using a rotating linear polarizer [6,7]. The first three Stokes parameters provide the degree of linear polarization $\mathrm{P}_{\mathrm{L}}$, from which the degree of circular polarization $\mathrm{P}_{\mathrm{C}}$ is obtained from $\mathrm{P}_{\mathrm{L}}^{2}+\mathrm{P}_{\mathrm{C}}^{2}=1$ assuming that no unpolarized $\mathrm{x}$ rays radiate from the EPU. Scans are shown corresponding to horizontal and vertical linear polarization, for which the PL2 was measured to be 0.98 and 0.99 , confirming this assumption to within the extinction ratio of the polarizer. The scan with the phase tuned to produce circular polarization yields $\mathrm{PC} 2=0.97$. Thus the polarization of $\mathrm{x}$-rays radiated by the EPU is just as designed, as expected and measured on similar devices.
Figure 3 also shows the net effects of organic contamination in the beamline. The brand new monochromator (first of this design) took longer to assemble than planned and accumulated some organic condensates. After 9 months of use there is a $50 \%$ dip from the finer grating, close to the usual high standards of UHV cleanliness at the ALS. The coarse grating was first in use and its flux dips at the carbon edge by a factor of three. This coarse grating has a high reflectivity nickel optical coating which may be oxidized by reactive oxygen cleaning techniques. We will use platinum or gold coatings in future.

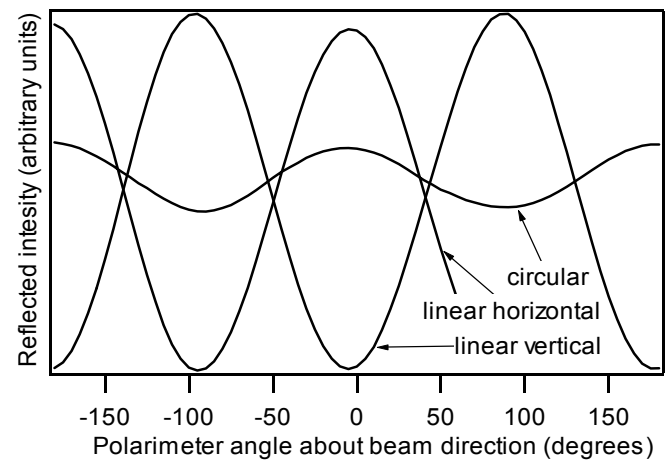

FIGURE 4. Polarimeter measurements to verify three polarization modes at $778 \mathrm{eV}$

\section{NEW ENGINEERING}

All the components in this beam line are new designs. The first mirror is a vertically collimating sagittal cylinder inside the shield wall. This will be one of a pair, each receiving light from one of the two undulators in the chicane straight. It has an internally cooled glidcop substrate $350 \mathrm{~mm}$ long with three adjustable angular degrees of freedom.

The monochromator has an aluminum chamber with explosion bonded stainless seal flanges and a 'helicoflex' seal under the lid. The beam enters and exits through the lid, and there is full access for alignment with the lid off. The entire monochromator moves transversely to the beam to illuminate different stripes on the single grating substrate, which is $50 \mathrm{~mm}$ wide. Water lines are carried along the sine-bars to minimize the use of couplings that flex during scanning and to avoid long bellows in UHV, which are hard to clean. All the internal parts (stainless steel and INVAR) are baked at $400^{\circ} \mathrm{F}$ in a vacuum furnace for cleanliness, prior to assembly.

Glass scale linear encoders are used on the external slides to read the angle of the optics. Compared to other implementations [8] that use internal rotary encoders, this is a potential source of error. We find that there is a detectable hysteresis (1:6000) in the relationship between encoder reading and true optic angle, caused by the slight strain in the drive components, which is quite reproducible, but depends on the scan direction. We scan always from low to high energy. Furthermore, small corrections are required to the geometrical energy calibration formula (6ev correction at $1800 \mathrm{eV}$ when calibrated at $400 \mathrm{eV}$ ). These calibration corrections are also quite reproducible and have been determined empirically. At this time we are still exploring the precision of the mechanism and its encoders. 


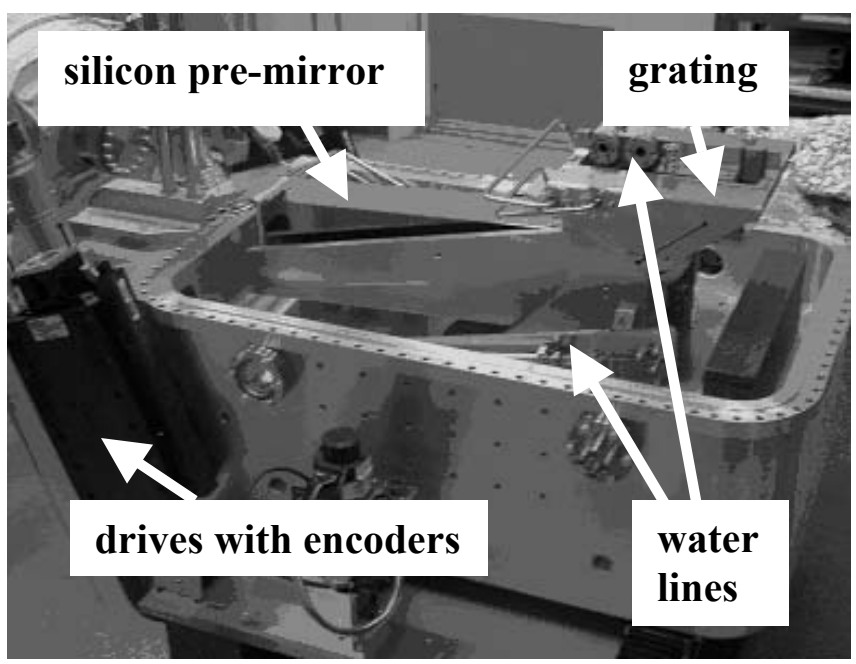

FIGURE 5. New monochromator during assembly of water lines.
The pre-mirror is an internally cooled silicon substrate $450 \mathrm{~mm}$ long, polished flat [9] with rms polished slope error $0.5 \mu \mathrm{rad}$ rms. The two grating stripes are ruled [10] on a single plane $(0.5 \mu \mathrm{rad} \mathrm{rms})$ 'glidcop' substrate $65 \mathrm{~mm} \mathrm{x}$ $160 \mathrm{~mm}$, to simplify the water connections. Actually this substrate is wide enough for up to 4 grating stripes, when used with a narrow undulator beam.

The toroidal focus mirrors are used interchangeably, one for each branch, each with its own set of exit slits. They are implemented as side cooled silicon substrates with a toroidal polished shape. The heat removal allows them to be used with zero order light at the spectral bandwidth of the undulator harmonic peak. One branch serves a diffraction limited zone plate

microscope that uses the (typically $50 \mu \mathrm{m}$ square) exit slit aperture as its illumination source. The other branch contains a pair of $\mathrm{KB}$ refocus mirrors that generate a $10 \mu \mathrm{m}$ spot in the spectroscopy experiments. This refocus is computer controlled and is described in a separate paper [11]. Changeover from one branch to the other requires only the motion of the first of these mirrors, into or out of the beam, and the selection of the appropriate grating stripe, thus we can switch between two fully aligned experiment set-ups in less than 30 seconds. This facility will run round the clock, six or seven days per week At the time of writing the commissioning of the beam line is not complete, further studies of undulator polarization and higher order components of the radiation are planned.

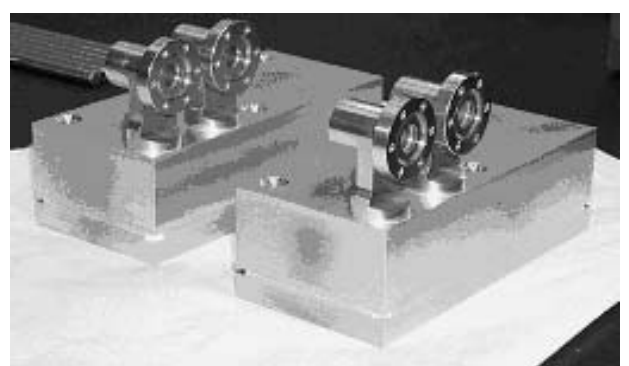

FIGURE 6. Glidcop grating substrates (only one is installed).

This project would not have been possible without the expertise of Mike DeCool and the vacuum group at the ALS. The Advanced Light Source and this work are supported by the Director, Office of Science, Office of Basic Energy Sciences, Division of Materials Sciences and the Division of Chemical Sciences, Geosciences, and Biosciences of the U.S. Department of Energy at Lawrence Berkeley National Laboratory under Contract No. DEAC03-76SF00098.

\section{REFERENCES}

1. H. Petersen, Optics Comm. 40 (1982) 402.

2. R. Follath and F. Senf, Nucl. Instr. Methods Phys. Res. A390 (1997) 388

3. D. Schmitz et. al. BESSY annual report 2002

4. T. Warwick et al, Nucl. Instr. Methods Phys. Res. A467-468 (2001) 525

5. T. Warwick and D. Shuh, MES project conceptual design, ALS Internal Report, LSBL 536

6. J. B. Kortright, M. Rice, and K. D. Frank, Rev. Sci. Instrum. 66, 1567 (1995).

7. R. Carr, J. B. Kortright, M. Rice, and S. Lidia, Rev. Sci. Instrum, 66, 1862 (1995).

8. Jen-Optic Mikrotechnik GmbH, Goschwitzer Strasse 40, D07745 Jena, Germany

9. InSync Inc., Broadbent Parkway, Albuquerque, New Mexico 87107, USA

10. Carl Zeiss Laser Optics, Carl-Zeiss-Strasse 22, D-73447 Oberkochen, Germany

11. T. Warwick et. al. "Large Aperture Micro-Focus KB Mirrors for Spectroscopy Experiments at the Advanced Light Source" Proc. 8th Intl. Conf Synchr. Rad. Instrum., San Francisco, California, August2003, American Inst. Physics. 